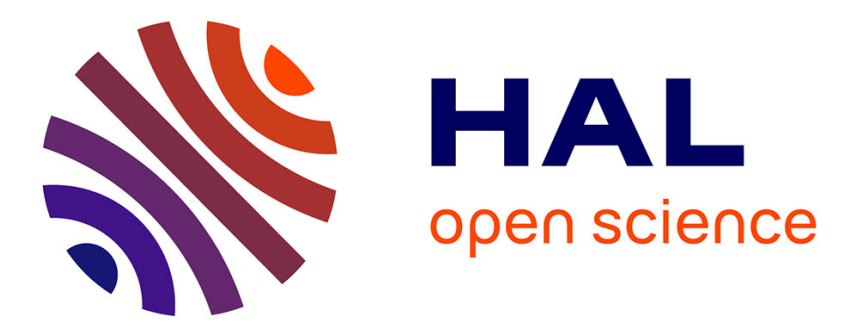

\title{
Plaques-boucles mérovingiennes de la fin du VIIe siècle Anna Vollgraff-Roes
}

\section{To cite this version:}

Anna Vollgraff-Roes. Plaques-boucles mérovingiennes de la fin du VIIe siècle. Gallia - Fouilles et monuments archéologiques en France métropolitaine, 1955, 13 (1), pp.97-99. 10.3406/galia.1955.1430 . hal-01923452

\section{HAL Id: hal-01923452 \\ https://hal.science/hal-01923452}

Submitted on 6 Feb 2020

HAL is a multi-disciplinary open access archive for the deposit and dissemination of scientific research documents, whether they are published or not. The documents may come from teaching and research institutions in France or abroad, or from public or private research centers.
L'archive ouverte pluridisciplinaire HAL, est destinée au dépôt et à la diffusion de documents scientifiques de niveau recherche, publiés ou non, émanant des établissements d'enseignement et de recherche français ou étrangers, des laboratoires publics ou privés.

\section{(이) $\$$}

Distributed under a Creative Commons Attribution - NonCommercial - NoDerivatives| 4.0 
la vie future n’étaient pas exprimées moins ouvertement, ni moins intimement mêlées à la trame de la routine quotidienne, que ne le sont la foi ot l'espoir des familles chrétiennes.

Jocelyn M.C. ToYNise.

Museum of Classical Archacolngy, Cambridge.

\section{Traduction P.-M. Duval.}

[Nous remercions vivement Miss ToYxBe, Professeur d'archéologie classique à l'Universile de Cambridge, de sa précicuse contribution aux problèmes soulevés par M. AYMand a propos de la belle découverte de vîmes. on ne manquera pas d'ètre frappé par le caractire si remarquable, a la fois original et somptueux, par le singulier intérèt et la date lardive des mosaïques britto-romaines. Et nous avons le plaisir d'annoncer qu'à l'occasion de l'enquête ouverte par l'Académie des Inscriptions en vue d'un Supplément à l'Inventaire des mosaïques de la Gaule, M. Henri STERn nous signale à Reims un nouveau pave-

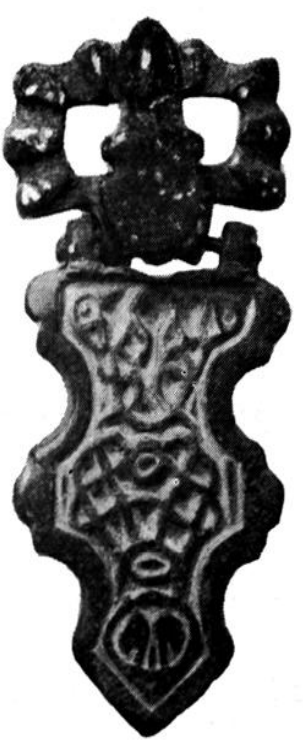

a

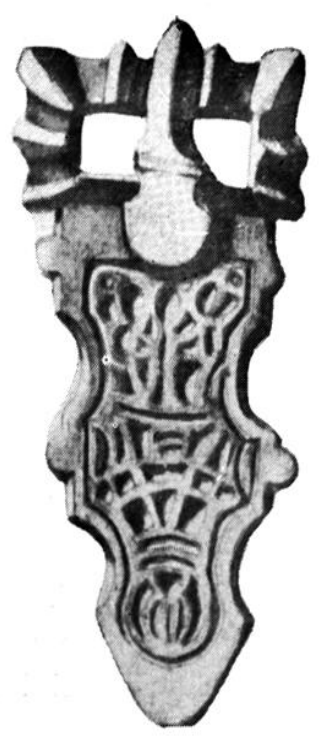

$\tau$

ment au Bellérophon, encore inédit, qui porte à huit le nombre de ces représentations du héros. Sept proviennent donc des régions occidentales, où se confirme ainsi le succès durable, ou, pour reprendre les mots de II. AYMARD, «la renaissance discrète mais lenace, aux $\mathrm{II}^{\mathrm{e}}$ et $\mathrm{III}^{\circ}$ siècles, de la légende du héros corinthien et lycien ».

P.-M. Duval.

\section{PlaQles-molgites MÉROVingienNes DE LA FIN IUU VII SIÈCLLE}

Ie Musée de Ireyde possc̀de une plaqueboucle de bronze (Iongueur: $8^{\mathrm{rm}}$ ), trouvée à Garderen (Gueldre), dont W. Pleyte. a déjà publié un dessin dans son grand ouvrage illustré sur les antiquités des Paỵs-Bas 1, mais qui n'a pas encore reçu l'attention qu'clle mérite (fig. 1a). Flle étail assujetlie sur le cuir, non pas par des bossettes mobiles, mais par des lan-

(1) W. PLEYTE, Nederlandsche Oudheden, Gelderland, pl. $\mathrm{xx}, 5$.

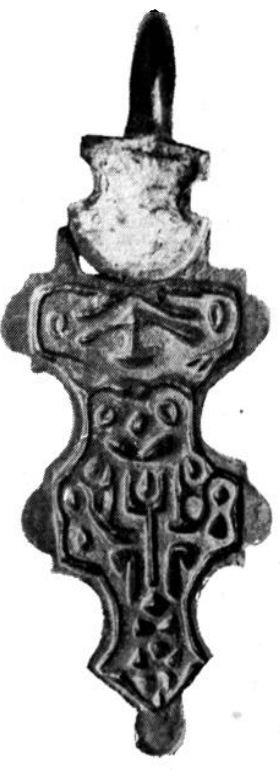

C

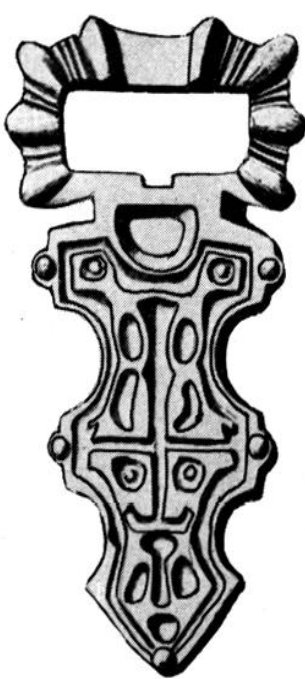

d

Fir. 1. -- Plaques-boucles de Garderen $(a)$, de l'Aisne $(b)$, du Musće de Rouen (c), de Monceaux (Oise). Haut. de la plaque a, sans la boucle : (j) moins de la moitis;. 
guettes fixes percées d'un trou. $\mathrm{Au}$ revers, le bord est légèrement surélevé.

C'est une pièce de forme insolite: les lignes sévères de l'anneau et du bord de la plaque, telles qu'elles prédominent à l'époque des migrations, sont remplacées ici par un contour mouvementé à l'excès, d'un style qu'on serait tenté d'appeler baroque. Le décor consiste en un lacis symétrique, auquel s'associent, tout en dessous, deux têtes d'oiseaux allongées, à becs dirigés vers le bas. En haut, deux points en relief entourés de cercles suggèrent une seconde paire de têtes, ornementation sans doute déjà plus ou moins abâtardie. Tout l'entrelacs laisse d'ailleur's une impression de dégénérescence : on parvient encore à en suivre les lignes dans leur cours sinueux, mais elles paraissent lourdes et incertaines, à les comparer avec d'autres exemplaires du même style (qu'on a coutume d'appeler le style II). L'objet appartient par conséquent à l'art mérovingien finissant, c'està-dire à la fin du vir siècle au plus tìt.

Dans le royaume des Pays-Bas, la plaque-boucle de Garderen est unique en son genre, mais elle a des parallèles dans la France du Nord-Ouest. L'analogie la plus frappante est fournie par une plaque-boucle reproduite dans un ouvrage de Pilloy 2, et probablement perdue (fig. 1b): Celle-ci représente, non un ctat antérieur mais, au contraire, un degré plus avancé dans l'évolution du thème. I.es traits que montre la plaqueboucle de Garderen sont encore là, mais ils sont, pour ainsi dire, géométrisés, et le modèle de l'entrelacs est faussé. I)ans la partie inférieure, les têtes d'oiseaux sont remplacées par quatre lignes parallèles; la bouclette qui les surmonte et qui est formée par l'entre-croisement de deux lignes du dessin original est réduite à deux encoches. Il en est de même pour une bouclette semblable placée un peu plus haut. I'ornementation n'est pas à proprement parler désagrégée : mais au lieu d'un entrelacs, on y croit voir un capricieux dessin de remplissage.

Dans la plaque-boucle (fig. 1c), que j'ai photographicée au muséc de Rouen, la décomposition du thème est chose accomplie. Le dícor y est scindé en deux parties, dont celle d'en bas (oì l'on remarque deux nouvelles têtes adventices) manque de cohésion. Sur une seconde plaque-boucle du même musée, malheureusement incomplète, le même motif survit sous une forme linéare; aux têtes d'oiseaux s'est substitué un losange inserit dans un carré (fig. 2).

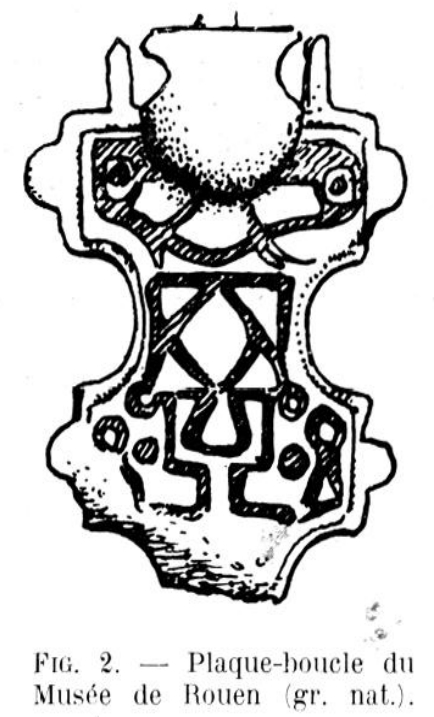

Dans la plaque-boucle (lig. 1d), qui provient de Monceaux (Oise)3, le dessin primordial s'est atrophici au point que seuls les rrilletons placés dans les angles supérieurs trahissent encore la dérivation du theme dócoratif d'un entrelacs du genre de relui de la fig. $1 a$.

(2) J. PILLoy, Etudes sur d'anciens lieux de sépulture dans l'Aisne, I, pl. v, 11. 20.
(3) Bull. arch. du Comité, 1908, pl. xxxı, 
Par un curieux effet du hasard, un type de plaque-boucle qui semblerait être originaire de la vallée de l'Aisne ou de celle de l'Oise, est représenté en $\mathrm{Hol}-$ lande par l'exemplaire le plus ancien connu. Je dis: l'exemplaire le plus ancien, et non : le prototype, car le décor de la plaque-boucle de Garderen ne rend apparemment pas la forme primitive du dessin.

Anne Roes.

Université d'Utrecht. 